\title{
Synthesis and Properties of Chiral Polyurethane Elastomers Using Tartaric Acids
}

\author{
Kazunori Kizuka*, Shin-Ichi Inoue \\ Applied Chemistry Department, Aichi Institute of Technology, Toyota, Japan \\ Email: *w13801ww@aitech.ac.jp
}

Received 18 November 2015; accepted 8 January 2016; published 12 January 2016

Copyright (C) 2016 by authors and Scientific Research Publishing Inc.

This work is licensed under the Creative Commons Attribution International License (CC BY). http://creativecommons.org/licenses/by/4.0/

c) (i) Open Access

\begin{abstract}
The polyaddition of isocyanate and polyol to form polyurethane elastomers has rarely been applied to the construction of chiral polyurethane elastomers. Hence, the introduction of chiral units via polyaddition remains a challenging subject in polymer chemistry. In this study, the synthesis of chiral polyurethane elastomers using an aromatic isocyanate, polyols (polyether and polyester polyols), and L(+)-, D(-)-, or meso-tartaric acid by a one-shot method is investigated. The polymers are characterized using FTIR and NMR spectroscopy, and their thermal properties are investigated by TGA, DMA, and DSC analyses. The optical activities of the polymers are confirmed by rotation. The use of chiral tartaric acids is essential to obtain the desired chiral polyurethane elastomers.
\end{abstract}

\section{Keywords}

Chiral, Polyurethane Elastomers, Tartaric Acid, Differential Scanning Calorimetry (DSC), Thermogravimetric Analysis (TGA)

\section{Introduction}

Polyurethane elastomers (PUEs) can be easily prepared by the polyaddition of isocyanate and polyol. Therefore, PUEs [1]-[7] are widely utilized for preparing flexible or rigid foams, leathers, coating materials, adhesives, sealants, elastomers, and fibers. PUEs derived from plants have been actively investigated in recent years because polymer materials using plants are expected to act as biodegradable replacements for materials derived from petroleum. Hence, we focused on the synthesis of PUEs containing natural products and the optical activity

*Corresponding author. 
of the obtained PUEs. The synthesis of chiral polymers is of paramount importance because of their special applications. The synthesis of chiral PUEs using chiral reagents, which are components of plants, was examined. There are three general ways to obtain optically active PUEs (i.e., either the pure left-handed or right-handed helical conformation or an excess of one of these conformations): 1) polyaddition of an optically inactive polyol with an isocyanate enantiomer [8]; 2) polyaddition of an optically inactive isocyanate with a polyol enantiomer [9]-[20]; and 3) polyaddition employing chiral additive reagents (e.g., chain extenders and cross-linkers) [21]. Method 2) has been used to prepare chiral PUEs; however, methods 1) and 3) have rarely been applied to the construction of optically active PUs. Tartaric acid is a stable biological molecule, which tastes like citric acid from citrus fruits. It is used as a raw material for cosmetics and medicines as well as in the industrial fields in fixing, plating, and plasticizing agents. Tartaric acid is a naturally occurring dicarboxylic acid containing two stereo-centers with identical substitution patterns. As it exists in nature, it is a racemic compound formed by dextrorotatory L-(+)-(2R, 3R) and levorotatory $\mathrm{D}(-)$-tartaric acid. The PUEs containing an achiral meso-tartaric acid (2R, 3S) can also be synthesized. Even today, investigations on macrocyclic [22]-[25] and complex compounds [26]-[30] using tartaric acids are frequently investigated. Recently, several studies on the synthesis of PUEs using tartaric acid have been reported [31] [32]. Various natural compounds including tartaric acid have been used as raw materials for PUE synthesis; these polymers have attracted considerable attention as a new biopolymer [33]-[40].

In this article, we report the synthesis of PUEs containing $\mathrm{L}(+)-, \mathrm{D}(-)$-, or meso-tartaric acid using an aromatic isocyanate, 4,4'-diphenylmethane diisocyanate (MDI), one of three different polyols (polytetramethylene glycol (PTMG), polycaprolactone diol (PCL), and polycarbonate diol (PCD)), and L(+)-, D(-)-, or meso-tartaric acid as a cross-linker via a one-shot method. The molecular structure analyses of these PUEs were conducted, and their chemical and physical properties were described. The appearance of asymmetry in PUEs containing $\mathrm{L}(+)-, \mathrm{D}(-)-$, or meso-tartaric acid was also investigated.

\section{Experimental}

\subsection{Materials}

PTMG (molecular weight = 2000; PTMG2000) was supplied by Invista Industry, Texas, USA. PCL (molecular weight = 2000; PCL2000) was supplied by Daicel Industry, Osaka, Japan. MDI and PCD (molecular weight = 2000; PCD2000) were supplied by Tosoh Industry, Tokyo, Japan (Tosoh). MDI was purified by distillation under reduced pressure $(267-400 \mathrm{~Pa})$ at $100^{\circ} \mathrm{C}$ before use. $\mathrm{L}(+)$ - and $\mathrm{D}(-)$-tartaric acid were purchased from Nacalai Tesque Inc., Kyoto, Japan (Nacalai) and used without further purification. Meso-tartaric acid was purchased from Tokyo Chemical Industry, Tokyo, Japan. Tetrahydrofuran (THF; Nacalai) was dried using $\mathrm{CaH}_{2}$ for several days and was distilled prior to use under an Ar atmosphere. Benzene was purchased from Nacalai. $N, N$ Dimethylformamide (DMF) (Nacalai) and dimethyl sulfoxide (DMSO) (Nacalai) were purchased and stored over $4 \AA$ molecular sieves before use. The following compounds were purchased from commercial suppliers and used as received: DMSO- $d_{6}$ (Euriso Top, Saint-Aubin, France), hexane (Nacalai), and acetone (Nacalai).

\subsection{Synthesis of Chiral Polyurethane Elastomers Containing Tartaric Acid}

Scheme 1 shows the preparation procedure for the L(+)-, D(-)-, or meso-tartaric-acid-containing PUEs. PUEs containing tartaric acid were prepared from MDI, a polyol (either PTMG2000, PCL2000, or PCD2000), and $\mathrm{L}(+)-, \mathrm{D}(-)-$, or meso-tartaric acid by a one-shot method. White solids separated out with increasing tartaric acid content. When the quantities of tartaric acid were $0.65 \mathrm{wt} \%, 1.4 \mathrm{wt} \%$, and $2.3 \mathrm{wt} \%$, the reaction time was 30 min. Conversely, when the quantities of tartaric acid were $3.4 \mathrm{wt} \%$ and $4.8 \mathrm{wt} \%$, the reaction time was $60 \mathrm{~min}$. The compositions of the PUEs are shown in Table 1. For example, the synthesis of PUE-PTMG-T1L can be described as follows. MDI (5.0 g, $\left.2.0 \times 10^{-2} \mathrm{~mol}\right)$, PTMG2000 (18 g, $\left.0.90 \times 10^{-2} \mathrm{~mol}\right), \mathrm{L}(-)$-tartaric acid $(0.34 \mathrm{~g}$, $\left.0.10 \times 10^{-2} \mathrm{~mol}\right)$, and THF $(20 \mathrm{~mL})$ were added to a $100 \mathrm{~mL}$ four-necked separable reaction flask equipped with a mechanical stirrer, gas inlet tube, and reflux condenser. The solution was then stirred at $100^{\circ} \mathrm{C}$ for 30 min under an Ar atmosphere.

\subsection{Preparation of Thin Films}

A sheet (thickness $=0.5-0.6 \mathrm{~mm}$ ) was obtained by casting the resulting solution $(20$ g). Sheets of PUE-PTMG- 


\section{+ $+\mathrm{HO}-\mathrm{R}-\mathrm{OH}+\mathrm{HO}-\mathrm{R}^{\prime}-\mathrm{OH}$ \\ OCN \\ 4,4'-diphenylmethane diisocyanate \\ Polyol}

(MDI)

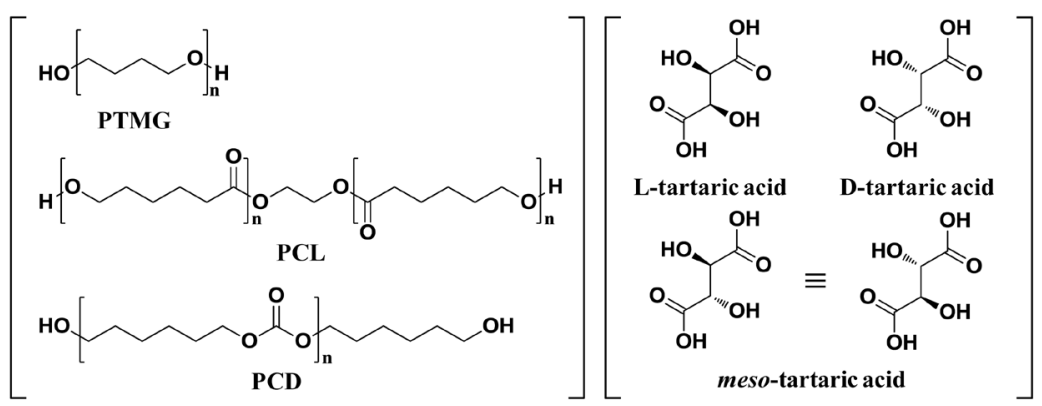

at $100{ }^{\circ} \mathrm{C}$, for $30-60 \mathrm{~min}$

PUE containing tartaric acid

in THF under Ar

Scheme 1. Preparation of the chiral polyurethane elastomers containing tartaric acid.

Table 1. Syntheses of chiral polyurethane elastomers containing $\mathrm{L}(+)-, \mathrm{D}(-)$-, and meso-tartaric acids.

\begin{tabular}{ccccc}
\hline Sample & MDI $\left(\mathrm{mol} \times 10^{-2}\right)$ & Polyol $^{\mathrm{a}}\left(\mathrm{mol} \times 10^{-2}\right)$ & Sucrose $\left(\mathrm{mol} \times 10^{-3}\right)$ & Sucrose content $(\mathrm{wt} \%)$ \\
\hline PUET1 (L/D/meso) & 2.0 & 0.90 & 0.10 & 0.65 \\
PUET2 (L/D/meso) & 2.0 & 0.80 & 0.20 & 1.4 \\
PUET3 (L/D/meso) & 2.0 & 0.70 & 0.30 & 2.3 \\
PUET4 (L/D/meso) & 2.0 & 0.60 & 0.40 & 3.4 \\
PUET5 (L/D/meso) & 2.0 & 0.50 & 0.50 & 4.8 \\
PUE & 2.0 & 1.0 & - & -
\end{tabular}

${ }^{\mathrm{a}}$ Polyol: polyoxytetramethylene glycol (molecular weight $\left.=2000\right)$, polycaprolactone diol (molecular weight $\left.=2000\right)$, polycarbonate diol $($ molecular weight $=2000$ )

T1-5 (L/D/meso) were formed using a disposable case at room temperature $\left(23^{\circ} \mathrm{C} \pm 2^{\circ} \mathrm{C}\right)$ for $15 \mathrm{~h}$ followed by treating at $100^{\circ} \mathrm{C}$ for $6 \mathrm{~h}$. In contrast, the sheets of PUE-PCL-T1-5 (L/D/meso) and PUE-PCD-T1-5 (L/D/meso) were formed using a disposable case at $100^{\circ} \mathrm{C}$ for $15 \mathrm{~h}$.

\subsection{Characterization}

All analyses and tests were performed at room temperature $\left(23^{\circ} \mathrm{C} \pm 2^{\circ} \mathrm{C}\right)$ unless otherwise indicated.

\subsubsection{Nuclear Magnetic Resonance (NMR) Spectroscopy}

${ }^{1} \mathrm{H}$ nuclear magnetic resonance (NMR; $\left.300 \mathrm{MHz}\right)$ and ${ }^{13} \mathrm{C}$ NMR $(75.4 \mathrm{MHz})$ spectra were recorded on a Varian Unity Plus-300 spectrometer using tetramethylsilane in dimethyl sulfoxide- $d_{6}$ (DMSO- $d_{6}$ ) at room temperature $\left(23^{\circ} \mathrm{C} \pm 2{ }^{\circ} \mathrm{C}\right)$ as an internal standard. The ${ }^{13} \mathrm{C}$ NMR acquisition parameters were as follows: spectral width $=$ $18.859 \mathrm{kHz}$; acquisition time $=1.816 \mathrm{~s}$; recycle delay $=0.185 \mathrm{~s}$; transients $=4000$; and spectral frequency $=75.4$ MHz.

\subsubsection{Fourier Transform Infrared (FTIR) Spectroscopy}

Fourier transform infrared (FTIR) spectra were recorded on a JASCO FT/IR-5300 using the attenuated total ref- 
lection (ATR) method and the transmission method at room temperature $\left(23^{\circ} \mathrm{C} \pm 2^{\circ} \mathrm{C}\right)$. The ATR spectrum was measured by an ATR500/M using an ATR KRS-5 prism.

\subsubsection{Gel Permeation Chromatography (GPC)}

The measurement conditions for GPC were as follows: sample, $0.1 \% N, N^{\prime}$-dimethylformamide (DMF) solution; solvent, DMF; column, TSK gels $\alpha-\mathrm{M}$ and TSK Gurdcolumn $\alpha$; flow rate, $500 \mu \mathrm{L} / \mathrm{min}$ at $40^{\circ} \mathrm{C}$; quantum, polystyrene transformation method.

\subsubsection{Chemical Properties}

Solubility tests were performed using $15 \times 15 \mathrm{~mm}$ test pieces. Each test piece was soaked in a solvent (benzene, hexane, acetone, THF, DMF, or DMSO; $8.0 \mathrm{~mL}$ ) at room temperature $\left(23^{\circ} \mathrm{C} \pm 2^{\circ} \mathrm{C}\right.$ ) or $100^{\circ} \mathrm{C}$ (for DMF and DMSO) for $24 \mathrm{~h}$.

Swelling tests were conducted using a test piece with dimensions of $15 \times 15 \mathrm{~mm}$. The degree of swelling (Rs) was calculated using the formula Rs $(\%)=W^{\prime} / W \times 100$, where $W^{\prime}$ is the weight of the test piece soaked in benzene for $24 \mathrm{~h}$, and $\mathrm{W}$ is the weight of the test piece after drying at $30^{\circ} \mathrm{C}$ for $24 \mathrm{~h}$ in vacuo.

\subsubsection{Mechanical Properties}

The hardness was investigated using a Kobunshi Keiki Asker Durometer (JIS A type) at room temperature $\left(23^{\circ} \mathrm{C} \pm 2^{\circ} \mathrm{C}\right)$. The test pieces for measurement were stacked up to a thickness of $6 \mathrm{~mm}$.

Tensile tests were conducted using an Orientec RTC-1225A with model-U-4300. A JIS 3-dumbel was used as the standard sample. The measurement conditions for the tensile tests were as follows: crosshead speed $=100$ $\mathrm{mm} / \mathrm{min}$ and room temperature $\left(23^{\circ} \mathrm{C} \pm 2^{\circ} \mathrm{C}\right)$. Tensile tests were performed on an Orientec RTC-1225A with a model-U-4300 using a JIS 3-dumbell as the standard sample and a crosshead speed of $100 \mathrm{~mm} / \mathrm{min}$.

\subsubsection{Thermal Properties}

Dynamic mechanical analysis (DMA) was performed on a Seiko Instruments DMS 6100 at a heating rate of $5^{\circ} \mathrm{C} / \mathrm{min}$ from $-100^{\circ} \mathrm{C}$ to $300^{\circ} \mathrm{C}$ and a frequency of $20 \mathrm{~Hz}$ under an $\mathrm{N}_{2}$ atmosphere.

Differential scanning calorimetry (DSC) measurements were performed on a Rigaku Thermo-Plus DSC-8230 at a heating rate of $10^{\circ} \mathrm{C} / \mathrm{min}$ from $-120^{\circ} \mathrm{C}$ to $200^{\circ} \mathrm{C}$ under an $\mathrm{Ar}$ atmosphere. Approximately $9.5 \mathrm{mg}$ of each composite was weighed and sealed in an aluminum pan. The samples were quickly cooled to $-120^{\circ} \mathrm{C}$ and then heated to $200^{\circ} \mathrm{C}$ at $10^{\circ} \mathrm{C} / \mathrm{min}$.

Thermogravimetric analysis (TGA) was performed with a Seiko Instruments TG/DTA6200 at a heating rate of $10^{\circ} \mathrm{C} / \mathrm{min}$ from $30^{\circ} \mathrm{C}$ to $500^{\circ} \mathrm{C}$ under an $\mathrm{N}_{2}$ atmosphere.

\subsubsection{Optical Rotation}

Optical rotation was measured by an Atago Polax-2L using a $200 \mathrm{~mm}$ observation pipe. The concentration of the sample was $10 \mathrm{wt} \%$ in $N$-methylpyrrolidone solution. The specific optical rotation was calculated using the following formula: $[\alpha]_{\mathrm{D}}=100 \alpha / \mathrm{L} \times \mathrm{C}$.

\subsubsection{Molecular Structure}

The molecular structures were calculated using Gaussian09W with the B3LYP/6-31G basis set. The molecular model was depicted using GaussView5.0.

\section{Results and Discussion}

\subsection{NMR Spectroscopy}

Analyses by ${ }^{1} \mathrm{H}$ NMR and ${ }^{13} \mathrm{C}$ NMR spectroscopy revealed that the polymers obtained were undoubtedly PUEs containing tartaric acid and that these polymers were composed from a urethane segment and tartaric acid. The NMR analyses indicated that tartaric acid was attached to the main PU chain as a cross-linker. For example, the ${ }^{1} \mathrm{H}$ NMR (DMSO- $d_{6}$ ) spectrum of PUE-PTMG-T1L included peaks at 8.55 ppm (-CO-NH-), 7.34 ppm and 7.08 ppm $\left(-\mathrm{C}_{6} \mathrm{H}_{4}-\right), 4.05 \mathrm{ppm}$ and $3.31 \mathrm{ppm}\left(-\mathrm{O}-\mathrm{CH}_{2}-\right), 3.78 \mathrm{ppm}\left(-\mathrm{C}_{6} \mathrm{H}_{4}-\mathrm{CH}_{2}-\mathrm{C}_{6} \mathrm{H}_{4}-\right)$, and $1.48 \mathrm{ppm}\left(-\mathrm{CH}_{2}-\mathrm{CH}_{2}-\right.$, -CH(R)-) (Figure 1). The ${ }^{13} \mathrm{C}$ NMR (DMSO- $d_{6}$ ) spectrum of PUE-PTMG-T1L contained peaks at $129 \mathrm{ppm}, 118$ ppm, 70 ppm, and 26 ppm (Figure 2). 


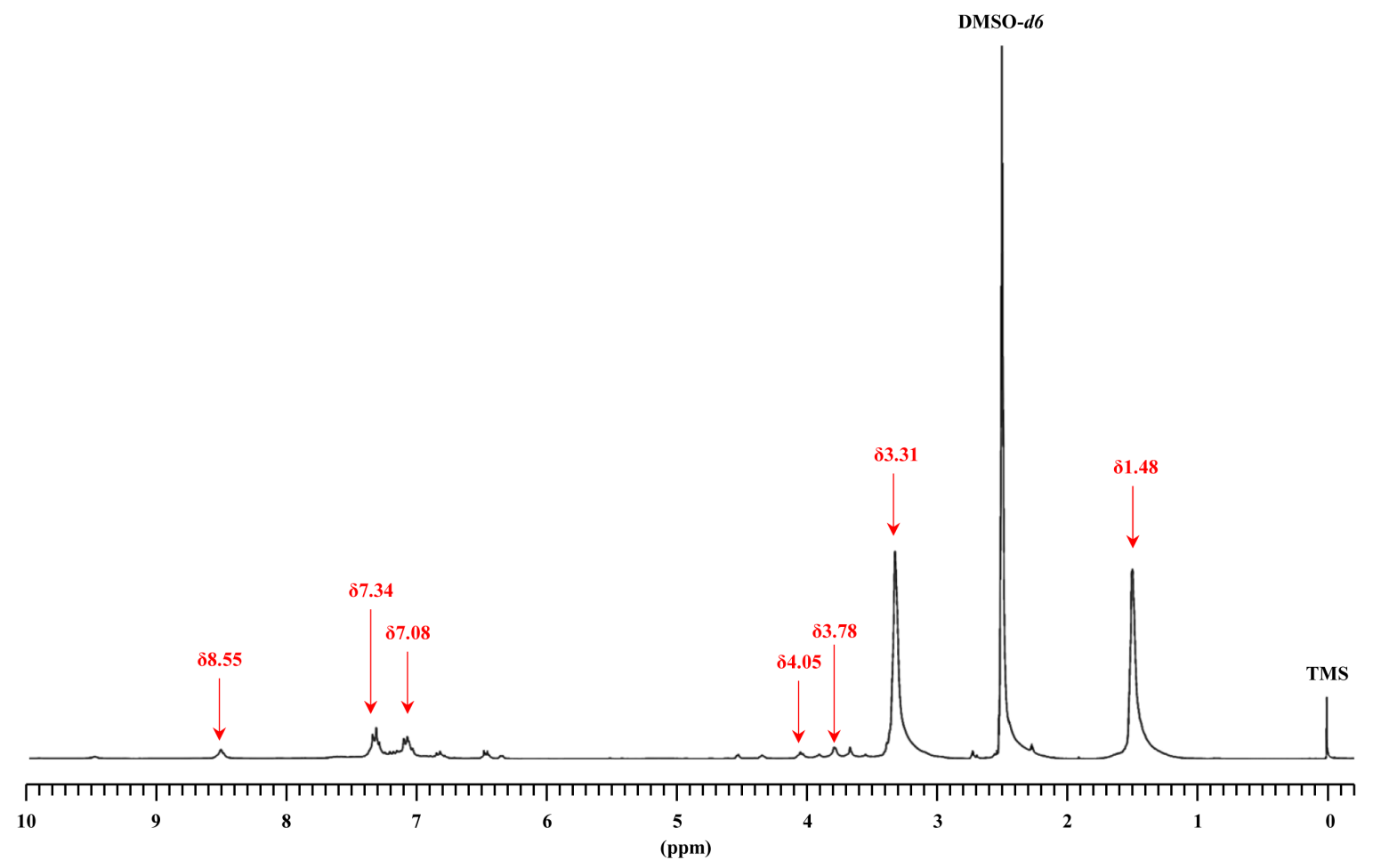

Figure 1. ${ }^{1} \mathrm{H}$ NMR spectrum of PUE-PTMG-TIL.

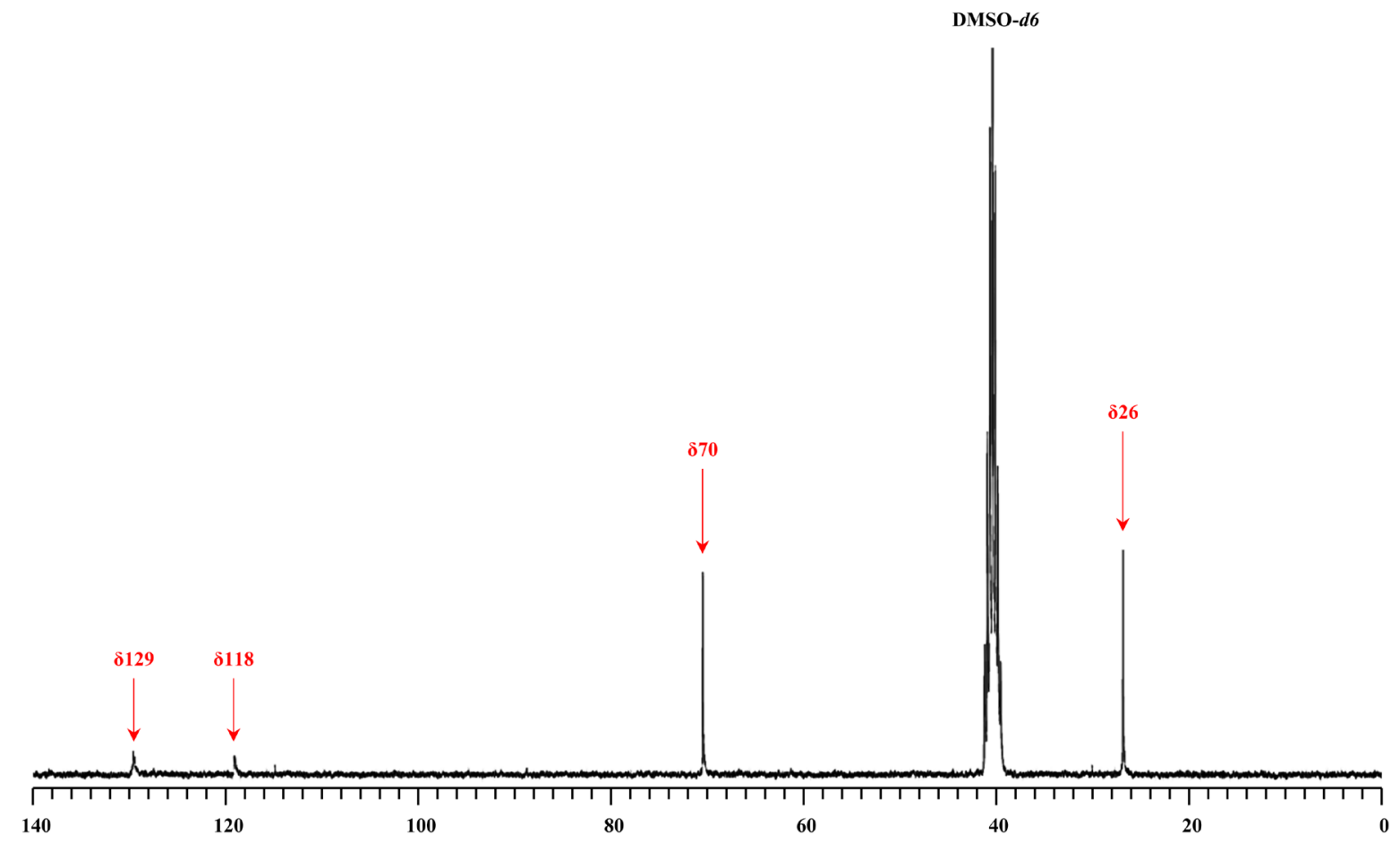

Figure 2. ${ }^{13} \mathrm{C}$ NMR spectrum of PUE-PTMG-TIL.

\subsection{FTIR Spectroscopy}

The IR spectra of PUE-PTMG-TL1-5 are shown in Figure 3. The band at $3292 \mathrm{~cm}^{-1}$ is related to the -NH of the 


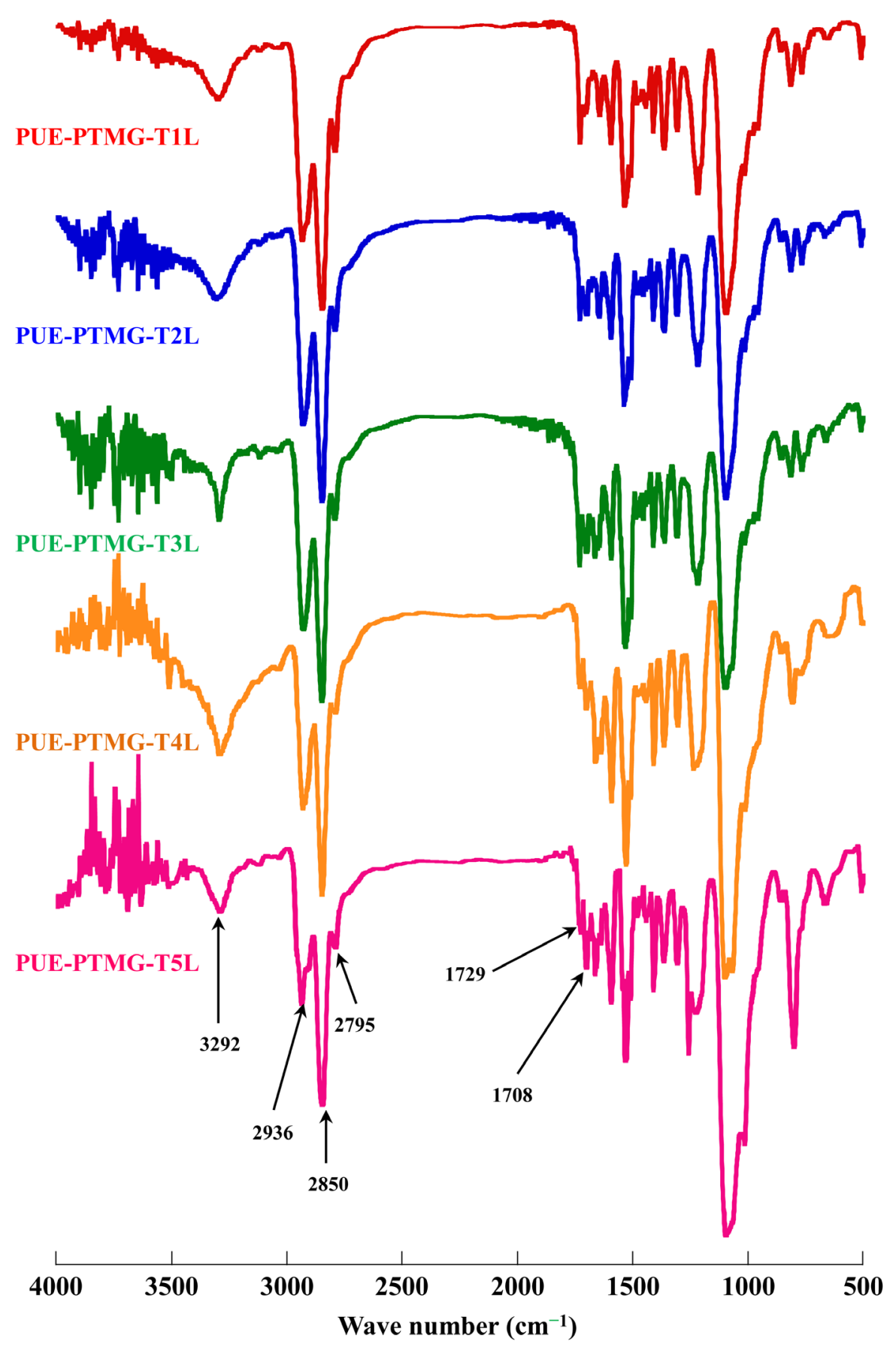

Figure 3. FT-IR (ATR method) spectra of PUE-PTMG-TI-5L.

polyurethane molecule, whereas the bands at $2936 \mathrm{~cm}^{-1}, 2850 \mathrm{~cm}^{-1}$, and $2795 \mathrm{~cm}^{-1}$ are attributable to the stretching vibration absorptions of aliphatic and aromatic -CH. The bands at $1729 \mathrm{~cm}^{-1}$ and $1708 \mathrm{~cm}^{-1}$ are related to the stretching vibration absorptions of $=\mathrm{CO}$ of urethane and the end of the polyurethane molecule, respectively.

\subsection{GPC}

The GPC of PUEs containing tartaric acid units is reported in Tables 2-4. For example, PUE-PTMG-TL1-5; Mw 47,000 - 150,000; Mw/Mn 2.2 - 6.1, PUE-PCL-TL1-5; Mw 34,000 - 85,000; Mw/Mn 2.8 - 7.2, PUE-PCD-TL1-5; 
Table 2. Physical properties of chiral PUEs containing L(+)-tartaric acid.

\begin{tabular}{|c|c|c|c|c|c|c|c|}
\hline Sample & $\begin{array}{l}\text { Hardness }^{\mathrm{a}} \\
\text { (JIS A) }\end{array}$ & $\begin{array}{c}\text { Swelling rate }^{\mathrm{b}} \\
(\%)\end{array}$ & {$[\alpha] \mathrm{D}^{\mathrm{c}}$} & $\begin{array}{l}T_{\mathrm{g}}^{\mathrm{d}} \\
\left({ }^{\circ} \mathrm{C}\right)\end{array}$ & $\begin{array}{l}T_{10}{ }^{\mathrm{e}} \\
\left({ }^{\circ} \mathrm{C}\right)\end{array}$ & $\begin{array}{l}M \mathrm{w}^{\mathrm{f}} \\
\left(\times 10^{4}\right)\end{array}$ & $M \mathrm{w} / M \mathrm{n}^{\mathrm{f}}$ \\
\hline PUE-PTMG-T1L & 80 & 240 & - & -67.1 & 355 & 15 & 3.3 \\
\hline PUE-PTMG-T2L & 82 & 231 & +2.5 & -65.1 & 352 & 10 & 2.4 \\
\hline PUE-PTMG-T3L & 84 & 222 & +4.2 & -68.8 & 350 & 7.4 & 2.2 \\
\hline PUE-PTMG-T4L & 86 & 190 & +5.0 & -67.8 & 338 & 6.6 & 4.2 \\
\hline PUE-PTMG-T5L & 87 & 174 & +5.8 & -67.7 & 338 & 4.7 & 6.1 \\
\hline PUE-PTMG & 77 & 229 & \pm 0.0 & -67.0 & 351 & 38 & 4.8 \\
\hline PUE-PCL-T1L & 78 & 200 & +0.50 & -46.7 & 349 & 8.5 & 2.8 \\
\hline PUE-PCL-T2L & 83 & 195 & +1.0 & -46.2 & 350 & 6.8 & 6.5 \\
\hline PUE-PCL-T3L & 88 & 182 & +2.2 & -44.6 & 347 & 4.8 & 5.9 \\
\hline PUE-PCL-T4L & 91 & 168 & +3.0 & -47.2 & 342 & 3.4 & 6.2 \\
\hline PUE-PCL-T5L & 94 & 148 & +5.2 & -48.0 & 334 & 3.4 & 7.2 \\
\hline PUE-PCL & 67 & 204 & \pm 0.0 & -45.0 & 338 & 16 & 3.5 \\
\hline PUE-PCD-T1L & 77 & 193 & - & -27.8 & 337 & 14 & 3.2 \\
\hline PUE-PCD-T2L & 83 & 189 & - & -28.6 & 336 & 9.4 & 3.3 \\
\hline PUE-PCD-T3L & 84 & 184 & +2.2 & -28.6 & 332 & 8.0 & 2.6 \\
\hline PUE-PCD-T4L & 88 & 175 & +3.0 & -28.5 & 330 & 6.1 & 2.3 \\
\hline PUE-PCD-T5 & 92 & 172 & +5.2 & -28.5 & 327 & 4.3 & 4.7 \\
\hline PUE-PCD & 79 & 195 & \pm 0.0 & -26.4 & 313 & 21 & 3.6 \\
\hline
\end{tabular}

${ }^{\mathrm{a}}$ Measurement conditions: JIS A type, total thickness $=6 \mathrm{~mm}$, measurement temperature $=$ room temperature $\left(23^{\circ} \mathrm{C} \pm 2{ }^{\circ} \mathrm{C}\right) .{ }^{\mathrm{b}} \mathrm{Measurement}$ conditions: solvent $=$ benzene, measurement temperature $=$ room temperature $\left(23^{\circ} \mathrm{C} \pm 2^{\circ} \mathrm{C}\right)$, measurement time $=24 \mathrm{~h} .{ }^{\mathrm{C}}$ Measurement conditions : solvent $=\mathrm{NMP}$, sample $=10 \mathrm{wt} \%$, measurement temperature $=$ room temperature $\left(23^{\circ} \mathrm{C} \pm 2{ }^{\circ} \mathrm{C}\right) .{ }^{\mathrm{d}}$ Differential scanning calorimetry performed at $10^{\circ} \mathrm{C} /$ min from $-120^{\circ} \mathrm{C}$ to $200^{\circ} \mathrm{C}$ under Ar atmosphere. ${ }^{\mathrm{e}}$ Thermogravimetric analysis performed at $10^{\circ} \mathrm{C} / \mathrm{min}$ from $30^{\circ} \mathrm{C}$ to $500^{\circ} \mathrm{C}$ under $\mathrm{N}_{2}$ atmosphere. ${ }^{\mathrm{f}} \mathrm{Measure}-$ ments conditions: solvent $=\mathrm{N}, \mathrm{N}$-dimethylformamide, sample $=0.1 \mathrm{wt} \%(\mathrm{~N}, \mathrm{~N}$-dimethylformamide/dimethyl sulfoxide $=1 / 1$ solution $)$, flow rate 500 $\mu \mathrm{L} / \mathrm{min}$, measurement temperature $=40^{\circ} \mathrm{C}$

Table 3. Physical properties of chiral PUEs containing D(-)-tartaric acid.

\begin{tabular}{|c|c|c|c|c|c|c|c|}
\hline Sample & $\begin{array}{l}\text { Hardness }^{\mathrm{a}} \\
\text { (JIS A) }\end{array}$ & $\begin{array}{c}\text { Swelling rate }{ }^{\mathrm{b}} \\
(\%)\end{array}$ & {$[\alpha] \mathrm{D}^{\mathrm{c}}$} & $\begin{array}{l}T_{\mathrm{g}}^{\mathrm{d}} \\
\left({ }^{\circ} \mathrm{C}\right)\end{array}$ & $\begin{array}{l}T_{10}{ }^{\mathrm{e}} \\
\left({ }^{\circ} \mathrm{C}\right)\end{array}$ & $\begin{array}{c}M w^{f} \\
\left(\times 10^{4}\right)\end{array}$ & $M \mathrm{w} / M \mathrm{n}^{\mathrm{f}}$ \\
\hline PUE-PTMG-T1D & 78 & 237 & - & -66.9 & 354 & 16 & 3.4 \\
\hline PUE-PTMG-T2D & 82 & 239 & -2.5 & -67.3 & 354 & 10 & 2.2 \\
\hline PUE-PTMG-T3D & 84 & 216 & -2.8 & -66.8 & 350 & 6.8 & 2.4 \\
\hline PUE-PTMG-T4D & 86 & 190 & -3.5 & -68.6 & 346 & 3.9 & 6.8 \\
\hline PUE-PTMG-T5D & 90 & 175 & -5.0 & -68.6 & 336 & 4.7 & 2.2 \\
\hline PUE-PTMG & 77 & 229 & \pm 0.0 & -67.0 & 351 & 38 & 4.8 \\
\hline PUE-PCL-T1D & 76 & 196 & -0.80 & -45.8 & 345 & 10 & 2.9 \\
\hline PUE-PCL-T2D & 86 & 191 & -2.0 & -47.6 & 349 & 5.7 & 7.6 \\
\hline PUE-PCL-T3D & 90 & 185 & -2.5 & -44.8 & 345 & 5.1 & 5.6 \\
\hline PUE-PCL-T4D & 92 & 165 & -3.8 & -46.8 & 338 & 2.6 & 8.3 \\
\hline
\end{tabular}




\section{Continued}

$\begin{array}{cccccccc}\text { PUE-PCL-T5D } & 92 & 150 & -5.5 & -47.1 & 338 & 3.2 & 6.3 \\ \text { PUE-PCL } & 67 & 204 & \pm 0.0 & -45.0 & 338 & 16 & 3.5 \\ \text { PUE-PCD-T1D } & 80 & 192 & - & -27.4 & 337 & 15 & 3.6 \\ \text { PUE-PCD-T2D } & 83 & 188 & - & -27.4 & 336 & 7.7 & 2.0 \\ \text { PUE-PCD-T3D } & 86 & 181 & -3.8 & -26.2 & 333 & 5.5 & 4.3 \\ \text { PUE-PCD-T4D } & 90 & 172 & -4.0 & -28.9 & 320 & 5.6 & 6.8 \\ \text { PUE-PCD-T5D } & 92 & 164 & -5.8 & -27.5 & 326 & 4.2 & 8.5 \\ \text { PUE-PCD } & 79 & 195 & \pm 0.0 & -26.4 & 313 & 21 & 3.6\end{array}$

${ }^{\mathrm{a}}$ Measurement conditions: JIS A type, total thickness $=6 \mathrm{~mm}$, measurement temperature $=$ room temperature $\left(23^{\circ} \mathrm{C} \pm 2^{\circ} \mathrm{C}\right) .{ }^{\mathrm{b}} \mathrm{Measurement}$ conditions: solvent $=$ benzene, measurement temperature $=$ room temperature $\left(23^{\circ} \mathrm{C} \pm 2{ }^{\circ} \mathrm{C}\right)$, measurement time $=24 \mathrm{~h}$. ${ }^{\mathrm{C}}$ Measurement conditions : solvent $=\mathrm{NMP}$, sample $=10 \mathrm{wt} \%$, measurement temperature $=$ room temperature $\left(23^{\circ} \mathrm{C} \pm 2{ }^{\circ} \mathrm{C}\right) .{ }^{d}$ Differential scanning calorimetry performed at $10^{\circ} \mathrm{C} /$ min from $-120^{\circ} \mathrm{C}$ to $200^{\circ} \mathrm{C}$ under Ar atmosphere. ${ }^{\mathrm{e}}$ Thermogravimetric analysis performed at $10^{\circ} \mathrm{C} / \mathrm{min}$ from $30^{\circ} \mathrm{C}$ to $500^{\circ} \mathrm{C}$ under $\mathrm{N}_{2}$ atmosphere. ${ }^{\mathrm{f}} \mathrm{Measure}-$ ments conditions: solvent $=\mathrm{N}, \mathrm{N}$-dimethylformamide, sample $=0.1 \mathrm{wt} \%(\mathrm{~N}, \mathrm{~N}$-dimethylformamide/dimethyl sulfoxide $=1 / 1$ solution), flow rate 500 $\mu \mathrm{L} / \mathrm{min}$, measurement temperature $=40^{\circ} \mathrm{C}$

\section{Table 4. Physical properties of chiral PUEs containing meso-tartaric acid.}

\begin{tabular}{|c|c|c|c|c|c|c|c|}
\hline Sample & $\begin{array}{l}\text { Hardness }^{\mathrm{a}} \\
\text { (JIS A) }\end{array}$ & $\begin{array}{c}\text { Swelling rate } \\
(\%)\end{array}$ & {$[\alpha] \mathrm{D}^{\mathrm{c}}$} & $\begin{array}{l}T_{\mathrm{g}}^{\mathrm{d}} \\
\left({ }^{\circ} \mathrm{C}\right)\end{array}$ & $\begin{array}{l}T_{10}{ }^{\mathrm{e}} \\
\left({ }^{\circ} \mathrm{C}\right)\end{array}$ & $\begin{array}{c}M \mathrm{w}^{\mathrm{f}} \\
\left(\times 10^{4}\right)\end{array}$ & $M w / M n^{f}$ \\
\hline PUE-PTMG-T1meso & 78 & 254 & \pm 0.0 & -67.4 & 350 & 13 & 3.2 \\
\hline PUE-PTMG-T2meso & 81 & 236 & \pm 0.0 & -67.4 & 354 & 9.8 & 2.9 \\
\hline PUE-PTMG-T3meso & 84 & 218 & \pm 0.0 & -67.9 & 349 & 7.6 & 8.2 \\
\hline PUE-PTMG-T4meso & 86 & 200 & \pm 0.0 & -68.1 & 344 & 5.7 & 2.3 \\
\hline PUE-PTMG-T5meso & 87 & 163 & \pm 0.0 & -67.7 & 348 & 3.9 & 7.5 \\
\hline PUE-PTMG & 77 & 229 & \pm 0.0 & -67.0 & 351 & 38 & 4.8 \\
\hline PUE-PCL-T1meso & 71 & 202 & \pm 0.0 & -45.5 & 345 & 14 & 4.8 \\
\hline PUE-PCL-T2meso & 83 & 198 & \pm 0.0 & -47.6 & 346 & 9.3 & 2.5 \\
\hline PUE-PCL-T3meso & 85 & 190 & \pm 0.0 & -47.5 & 345 & 8.6 & 2.5 \\
\hline PUE-PCL-T4meso & 88 & 178 & \pm 0.0 & -48.7 & 338 & 6.8 & 2.4 \\
\hline PUE-PCL-T5meso & 89 & 162 & \pm 0.0 & -45.2 & 325 & 4.4 & 2.1 \\
\hline PUE-PCL & 67 & 204 & \pm 0.0 & -45.0 & 338 & 16 & 3.5 \\
\hline PUE-PCD-T1meso & 80 & 190 & \pm 0.0 & -30.1 & 336 & 20 & 5.0 \\
\hline PUE-PCD-T2meso & 83 & 187 & \pm 0.0 & -29.7 & 335 & 11 & 3.2 \\
\hline PUE-PCD-T3meso & 84 & 182 & \pm 0.0 & -30.6 & 333 & 6.4 & 2.8 \\
\hline PUE-PCD-T4meso & 87 & 178 & \pm 0.0 & -30.7 & 340 & 6.1 & 2.8 \\
\hline PUE-PCD-T5meso & 90 & 163 & \pm 0.0 & -28.0 & 338 & 4.4 & 2.6 \\
\hline PUE-PCD & 79 & 195 & \pm 0.0 & -26.4 & 313 & 21 & 3.6 \\
\hline
\end{tabular}

${ }^{\mathrm{a}}$ Measurement conditions: JIS A type, total thickness $=6 \mathrm{~mm}$, measurement temperature $=$ room temperature $\left(23^{\circ} \mathrm{C} \pm 2^{\circ} \mathrm{C}\right) .{ }^{\mathrm{b}} \mathrm{Measurement}$ conditions: solvent $=$ benzene, measurement temperature $=$ room temperature $\left(23^{\circ} \mathrm{C} \pm 2^{\circ} \mathrm{C}\right)$, measurement time $=24 \mathrm{~h}$. ${ }^{\mathrm{c}}$ Measurement conditions : solvent $=\mathrm{NMP}$, sample $=10 \mathrm{wt} \%$, measurement temperature $=$ room temperature $\left(23^{\circ} \mathrm{C} \pm 2{ }^{\circ} \mathrm{C}\right) .{ }^{\mathrm{d}}$ Differential scanning calorimetry performed at $10^{\circ} \mathrm{C} / \mathrm{min}$ from $-120^{\circ} \mathrm{C}$ to $200^{\circ} \mathrm{C}$ under Ar atmosphere. ${ }^{\mathrm{e}}$ Thermogravimetric analysis performed at $10^{\circ} \mathrm{C} / \mathrm{min}$ from $30^{\circ} \mathrm{C}$ to $500^{\circ} \mathrm{C}$ under $\mathrm{N}_{2}$ atmosphere. ${ }^{\mathrm{f}} \mathrm{Measure}-$ ments conditions: solvent $=N, N$-dimethylformamide, sample $=0.1 \mathrm{wt} \%(\mathrm{~N}, \mathrm{~N}$-dimethylformamide/dimethyl sulfoxide $=1 / 1$ solution $)$, flow rate 500 $\mu \mathrm{L} / \mathrm{min}$, measurement temperature $=40^{\circ} \mathrm{C}$. 
Mw 43,000 - 140,000; Mw/Mn 2.6 - 4.7. The results indicate that the molecular weights of PUEs with tartaric acid decrease with increasing tartaric acid content in the composite.

\subsection{Chemical Properties}

The solvent resistances of the chiral tartaric-acid-containing PUEs were investigated. The sheets were immersed in various organic solvents (hexane, toluene, acetone, THF, DMF, and DMSO) for $24 \mathrm{~h}$. The results are shown in Table 5. None of the sheets changed at all in acetone and hexane at room temperature $\left(23^{\circ} \mathrm{C} \pm 2^{\circ} \mathrm{C}\right)$, but all swelled in benzene, THF, DMF, and DMSO. The sheets dissolved in DMF and DMSO at $100^{\circ} \mathrm{C}$. Thus, the obtained chiral PUEs containing tartaric acid showed good solvent resistances.

\subsection{Mechanical Properties}

The results of the hardness test for the PUEs containing $\mathrm{L}(+)-, \mathrm{D}(-)$-, and meso-tartaric acid are shown in Tables 2-4, respectively. The results indicate that the hardness increases with increasing tartaric acid content in the composite.

The tensile properties of the $\mathrm{L}(+)-, \mathrm{D}(-)-$, and meso-tartaric-acid-containing PUEs are reported in Tables 6-8, respectively. The tensile strengths of PUE-(PTMG/PCL)-T1(L/D), PUE-(PTMG/PCL/PCD)-T1 and 2(meso), and PUE-PCL-T1(meso) were greater and the tensile strengths of PUE-(PTMG/PCL)-T2-5(L/D), PUE-PCL-T25(meso), and PUE-(PTMG/PCL/PCD)-T3-5(meso) were smaller than those of the PUEs without tartaric acid. The tensile strengths of PUEs with tartaric acid decreased with increasing tartaric acid content. The elongation at the breaking points of PUE-(PTMG/PCL)-T1-3(L/D), PUE-PCL-T4(meso), PUE-PCD-T1-4 (L/D/meso), and PUE-PCD-T5(L) were greater and the elongation at the breaking points of PUE-(PTMG/PCL)-T4 and 5(L/D), PUE-PCL-T5(D) and PUE-PCD-T5(meso) were smaller than those of the PUEs without tartaric acid. The mixture of the tartaric acid and polyurethane chains in the soft amorphous phases reduced the mobility of the macromolecular chains, thereby generating stiff PUEs with tartaric acid when tartaric acid was in the matrix phase. Tartaric acid is a rigid, whereas polyurethane is a ductile elastomer. As a result, the tensile strengths and elongation at the breaking points of PUE-(PTMG/PCL/PCD)-T1 (L/D/meso) were better than those of the PUEs without tartaric acid. In addition, the comprehensive mechanical properties of the PUEs containing tartaric acid were superior to those of the PUEs without tartaric acid content, as shown in Figure 4.

The addition of tartaric acid has a wide range of effects when its content is over $1.4 \mathrm{wt} \%$. The initial modulus increases with increasing tartaric acid content in the composite. The PUE calcifies upon the addition of tartaric acid. The PUE with $0.65 \mathrm{wt} \%$ tartaric acid content showed the best elastomeric behavior.

Agreement between the results of the tensile and hardness tests suggests that the network chain density in the composites increases with increasing tartaric acid content.

Table 5. Solubility test results for chiral PUEs containing L(+)-, D(-)-, and meso-tartaric acids.

\begin{tabular}{|c|c|c|c|c|c|c|c|}
\hline \multirow{2}{*}{ Sample $e^{\mathrm{a}, \mathrm{b}}$} & \multirow{2}{*}{ Benzene $^{c}$} & \multirow{2}{*}{ Hexane $^{c}$} & \multirow{2}{*}{ THF $^{\mathrm{c}}$} & \multicolumn{2}{|c|}{$\mathrm{DMF}^{\mathrm{d}}$} & \multicolumn{2}{|c|}{$\mathrm{DMSO}^{\mathrm{d}}$} \\
\hline & & & & $23^{\circ} \mathrm{C}$ & $100^{\circ} \mathrm{C}$ & $23^{\circ} \mathrm{C}$ & $100^{\circ} \mathrm{C}$ \\
\hline PUE-Polyol-T1 & $\Delta$ & $x$ & $x$ & $\Delta$ & $\Delta$ & $\circ$ & $\Delta$ \\
\hline PUE-Polyol-T2 & $\Delta$ & $x$ & $x$ & $\Delta$ & $\Delta$ & $\circ$ & $\Delta$ \\
\hline PUE-Polyol-T3 & $\Delta$ & $x$ & $x$ & $\Delta$ & $\Delta$ & $\circ$ & $\Delta$ \\
\hline PUE-Polyol-T4 & $\Delta$ & $x$ & $x$ & $\Delta$ & $\Delta$ & $\circ$ & $\Delta$ \\
\hline PUE-Polyol-T5 & $\Delta$ & $x$ & $x$ & $\Delta$ & $\Delta$ & $\circ$ & $\Delta$ \\
\hline PUE-Polyol & $\Delta$ & $x$ & $\Delta$ & $\Delta$ & $\Delta$ & ○ & $\Delta$ \\
\hline
\end{tabular}

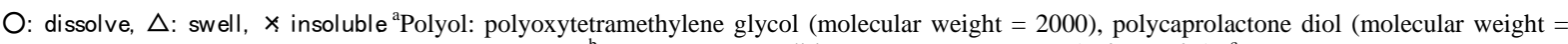
$2000)$, polycarbonate diol (molecular weight $=2000)$. ${ }^{\mathrm{b}}$ Measurement conditions: room temperature $\left(23^{\circ} \mathrm{C} \pm 2{ }^{\circ} \mathrm{C}\right) .{ }^{\mathrm{c}} \mathrm{Measurement}$ conditions: room temperature $\left(23^{\circ} \mathrm{C} \pm 2^{\circ} \mathrm{C}\right)$ and $100^{\circ} \mathrm{C}$. 
Table 6. Tensile properties of chiral PUEs containing L(+)-tartaric acid.

\begin{tabular}{cccccccccccc}
\hline Sample & M10 & $\begin{array}{c}\text { M50 } \\
(\mathrm{MPa})\end{array}$ & $\begin{array}{c}\text { M100 } \\
(\mathrm{MPa})\end{array}$ & $\begin{array}{c}\text { M200 } \\
(\mathrm{MPa})\end{array}$ & $\begin{array}{c}\text { M300 } \\
(\mathrm{MPa})\end{array}$ & $\begin{array}{c}\text { M400 } \\
(\mathrm{MPa})\end{array}$ & $\begin{array}{c}\text { M500 } \\
(\mathrm{MPa})\end{array}$ & $\begin{array}{c}\text { M600 } \\
(\mathrm{MPa})\end{array}$ & $\begin{array}{c}\text { M700 } \\
(\mathrm{MPa})\end{array}$ & $\begin{array}{c}\mathrm{TB}^{\mathrm{b}} \\
(\mathrm{MPa})\end{array}$ & $\begin{array}{c}\mathrm{EB}^{\mathrm{c}} \\
(\%)\end{array}$ \\
\hline PUE-PTMG-T1L & 2.38 & 4.13 & 4.86 & 6.62 & 10.5 & 17.7 & 32.1 & 55.5 & - & 59.9 & 615 \\
PUE-PTMG-T2L & 2.47 & 4.36 & 5.32 & 7.41 & 11.0 & 16.3 & 24.2 & 33.4 & 43.8 & 45.6 & 715 \\
PUE-PTMG-T3L & 2.31 & 4.21 & 5.20 & 7.34 & 10.6 & 14.7 & 19.7 & 24.9 & - & 28.9 & 677 \\
PUE-PTMG-T4L & 3.31 & 5.76 & 6.79 & 8.68 & 10.2 & 11.5 & - & - & - & 12.2 & 452 \\
PUE-PTMG-T5L & 4.01 & 6.70 & - & - & - & - & - & - & - & 7.37 & 91.0 \\
PUE-PTMG & 2.07 & 3.43 & 4.13 & 4.78 & 5.68 & 17.7 & 36.8 & - & - & 55.0 & 551 \\
PUE-PCL-T1L & 2.07 & 3.25 & 2.37 & 7.26 & 14.7 & 26.0 & 56.2 & - & - & 61.3 & 509 \\
PUE-PCL-T2L & 3.31 & 4.52 & 3.95 & 9.47 & 15.1 & 21.7 & 30.8 & - & - & 45.2 & 590 \\
PUE-PCL-T3L & 6.55 & 7.27 & 5.44 & 11.2 & 16.0 & 21.8 & 28.2 & - & - & 35.3 & 582 \\
PUE-PCL-T4L & 7.27 & 7.95 & 7.76 & 12.4 & 15.5 & 18.4 & - & - & - & 18.4 & 401 \\
PUE-PCL-T5L & 7.96 & 9.40 & 8.64 & - & - & - & - & - & - & 10.5 & 109 \\
PUE-PCL & 1.37 & 2.74 & 3.39 & 6.49 & 17.8 & - & - & - & - & 49.1 & 398 \\
PUE-PCD-T1L & 2.51 & 4.35 & 5.89 & 15.4 & 52.0 & - & - & - & - & 66.0 & 398 \\
PUE-PCD-T2L & 3.17 & 5.66 & 8.17 & 18.5 & 41.3 & - & - & - & - & 60.7 & 320 \\
PUE-PCD-T3L & 4.32 & 7.58 & 10.6 & 20.7 & 38.7 & - & - & - & - & 55.1 & 352 \\
PUE-PCD-T4L & 5.61 & 9.73 & 13.2 & 23.2 & 36.5 & - & - & - & - & 39.2 & 371 \\
PUE-PCD-T5L & 7.09 & 11.5 & 14.4 & 23.7 & - & - & - & - & - & 29.0 & 326 \\
PUE-PCD & 1.25 & 2.87 & 3.95 & 11.9 & - & - & - & - & - & 58.9 & 273 \\
\hline
\end{tabular}

${ }^{\mathrm{a}}$ Tensile properties measured at room temperature $\left(23^{\circ} \mathrm{C} \pm 2^{\circ} \mathrm{C}\right)$ with strain speed of $100 \mathrm{~mm} / \mathrm{min}$. ${ }^{\mathrm{b}} \mathrm{Tensile}$ strength at breaking point. ${ }^{\mathrm{c}}$ Elongation at breaking point.

Table 7. Tensile properties of chiral PUEs containing D(-)-tartaric acid.

\begin{tabular}{cccccccccccc}
\hline Sample & $\begin{array}{c}\text { M10 } \\
(\mathrm{MPa})\end{array}$ & $\begin{array}{c}\text { M50 } \\
(\mathrm{MPa})\end{array}$ & $\begin{array}{c}\text { M100 } \\
(\mathrm{MPa})\end{array}$ & $\begin{array}{c}\text { M200 } \\
(\mathrm{MPa})\end{array}$ & $\begin{array}{c}\text { M300 } \\
(\mathrm{MPa})\end{array}$ & $\begin{array}{c}\text { M400 } \\
(\mathrm{MPa})\end{array}$ & $\begin{array}{c}\text { M500 } \\
(\mathrm{MPa})\end{array}$ & $\begin{array}{c}\text { M600 } \\
(\mathrm{MPa})\end{array}$ & $\begin{array}{c}\text { M700 } \\
(\mathrm{MPa})\end{array}$ & $\begin{array}{c}\mathrm{TB}^{\mathrm{b}} \\
(\mathrm{MPa})\end{array}$ & $\begin{array}{c}\mathrm{EB}^{\mathrm{c}} \\
(\%)\end{array}$ \\
\hline PUE-PTMG-T1D & 4.63 & 5.39 & 6.09 & 8.15 & 12.2 & 18.5 & 30.7 & 47.3 & - & 55.7 & 637 \\
PUE-PTMG-T2D & 2.75 & 4.75 & 5.61 & 7.32 & 10.1 & 13.8 & 18.7 & 25.0 & 31.0 & 38.7 & 819 \\
PUE-PTMG-T3D & 3.77 & 6.07 & 7.00 & 8.84 & 11.1 & 13.4 & 16.0 & 19.2 & 21.6 & 22.7 & 740 \\
PUE-PTMG-T4D & 3.73 & 5.89 & 6.78 & 8.23 & 9.09 & - & - & - & - & 9.12 & 307 \\
PUE-PTMG-T5D & 4.94 & 7.75 & 8.57 & - & - & - & - & - & - & 9.10 & 152 \\
PUE-PTMG & 2.07 & 3.43 & 4.13 & 4.78 & 5.68 & 17.7 & 36.8 & - & - & 55.0 & 551 \\
PUE-PCL-T1D & 1.62 & 2.70 & 3.34 & 6.04 & 12.3 & 20.8 & 36.3 & - & - & 57.5 & 564 \\
PUE-PCL-T2D & 5.42 & 6.48 & 7.25 & 11.3 & 16.0 & 21.6 & 28.4 & 39.2 & - & 42.5 & 625 \\
PUE-PCL-T3D & 6.01 & 7.25 & 8.07 & 12.0 & 16.1 & 20.5 & 25.4 & 30.7 & - & 32.6 & 627 \\
PUE-PCL-T4D & 6.67 & 7.91 & 8.76 & 12.1 & 14.9 & - & - & - & - & 16.7 & 388 \\
PUE-PCL-T5D & 9.00 & - & - & - & - & - & - & - & - & 9.56 & 23.5 \\
PUE-PCL & 1.37 & 2.74 & 3.39 & 6.49 & 17.8 & - & - & - & - & 49.1 & 398 \\
PUE-PCD-T1D & 2.71 & 4.92 & 7.53 & 20.1 & 52.3 & - & - & - & - & 70.1 & 331 \\
PUE-PCD-T2D & 3.14 & 5.65 & 8.18 & 19.2 & 45.6 & - & - & - & - & 65.1 & 345 \\
PUE-PCD-T3D & 4.08 & 6.97 & 9.37 & 18.8 & 38.7 & - & - & - & - & 53.3 & 352 \\
PUE-PCD-T4D & 5.15 & 9.02 & 12.4 & 21.6 & 35.3 & - & - & - & - & 36.7 & 311 \\
PUE-PCD-T5D & 6.42 & 11.0 & 14.5 & 23.7 & - & - & - & - & - & 29.1 & 266 \\
PUE-PCD & 1.25 & 2.87 & 3.95 & 11.9 & - & - & - & - & - & 58.9 & 273 \\
\hline
\end{tabular}

${ }^{\mathrm{a}}$ Tensile properties measured at room temperature $\left(23^{\circ} \mathrm{C} \pm 2^{\circ} \mathrm{C}\right)$ with strain speed of $100 \mathrm{~mm} / \mathrm{min}$. ${ }^{\mathrm{b}}$ Tensile strength at breaking point. ${ }^{\mathrm{c}}$ Elongation at breaking point. 


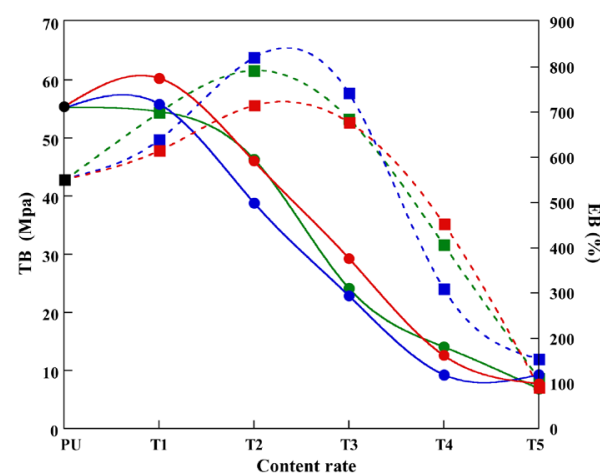

(a)

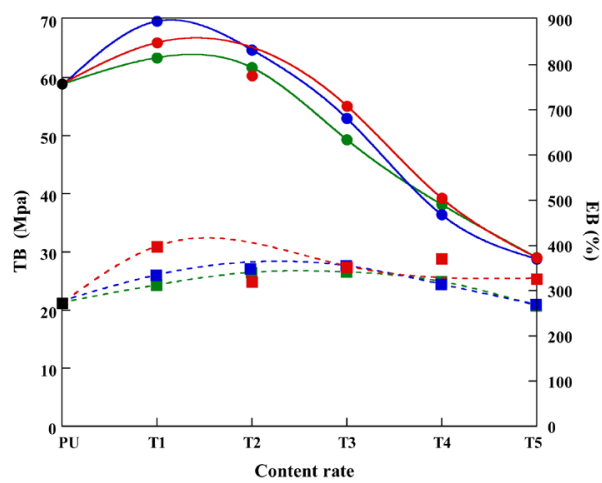

(c)

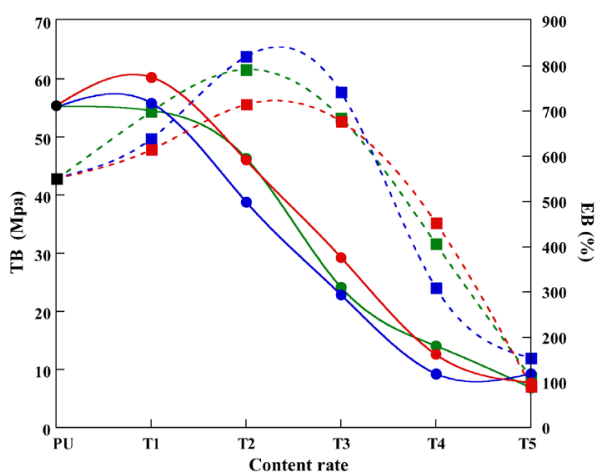

(b)

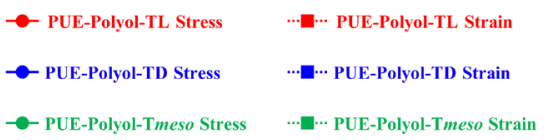

Figure 4. The tensile strengths and elongation at the breaking points for PUEs with introducing of the tartaric acid. (a) PUE-PTMG and PUE-PTMG-TI-5 (L/D/meso); (b) PUE-PCL and PUE-PCL-TI-5 (L/D/meso); and (c) PUE-PCD and PUE-PCD-TI-5 (L/D/meso).

Table 8. Tensile properties of chiral PUEs containing meso-tartaric acid.

\begin{tabular}{cccccccccccc}
\hline Sample $^{\mathrm{a}}$ & $\begin{array}{c}\text { M10 } \\
(\mathrm{MPa})\end{array}$ & $\begin{array}{c}\text { M50 } \\
(\mathrm{MPa})\end{array}$ & $\begin{array}{c}\text { M100 } \\
(\mathrm{MPa})\end{array}$ & $\begin{array}{c}\text { M200 } \\
(\mathrm{MPa})\end{array}$ & $\begin{array}{c}\text { M300 } \\
(\mathrm{MPa})\end{array}$ & $\begin{array}{c}\text { M400 } \\
(\mathrm{MPa})\end{array}$ & $\begin{array}{c}\text { M500 } \\
(\mathrm{MPa})\end{array}$ & $\begin{array}{c}\text { M600 } \\
(\mathrm{MPa})\end{array}$ & $\begin{array}{c}\text { M700 } \\
(\mathrm{MPa})\end{array}$ & $\begin{array}{c}\mathrm{TB}^{\mathrm{b}} \\
(\mathrm{MPa})\end{array}$ & $\begin{array}{c}\mathrm{EB}^{\mathrm{c}} \\
(\%)\end{array}$ \\
\hline PUE-PTMG-T1meso & 2.11 & 3.68 & 4.51 & 6.33 & 9.74 & 15.5 & 26.1 & 38.0 & 54.0 & 54.3 & 702 \\
PUE-PTMG-T2meso & 4.09 & 6.82 & 7.91 & 1.00 & 12.8 & 16.9 & 22.5 & 3.04 & 38.2 & 46.1 & 795 \\
PUE-PTMG-T3meso & 3.92 & 6.22 & 7.27 & 9.33 & 11.8 & 14.4 & 17.3 & 21.1 & - & 23.9 & 688 \\
PUE-PTMG-T4meso & 4.13 & 7.09 & 8.42 & 10.4 & 12.5 & 13.8 & - & - & - & 13.8 & 409 \\
PUE-PTMG-T5meso & 3.49 & 5.68 & 6.49 & - & - & - & - & - & - & 6.61 & 113 \\
PUE-PTMG & 2.07 & 3.43 & 4.13 & 4.78 & 5.68 & 17.7 & 36.8 & - & - & 55.0 & 551 \\
PUE-PCL-T1meso & 3.61 & 4.61 & 5.11 & 8.59 & 15.0 & 24.6 & 46.2 & - & - & 62.3 & 539 \\
PUE-PCL-T2meso & 5.77 & 6.34 & 7.00 & 10.5 & 15.0 & 20.6 & 27.0 & 37.5 & - & 40.4 & 624 \\
PUE-PCL-T3meso & 5.50 & 6.13 & 6.78 & 10.3 & 14.2 & 18.7 & 24.6 & - & - & 27.7 & 558 \\
PUE-PCL-T4meso & 5.62 & 6.55 & 7.48 & 10.8 & 14.3 & 17.5 & - & - & - & 17.8 & 411 \\
PUE-PCL-T5meso & 4.54 & 6.09 & 7.28 & 10.7 & - & - & - & - & - & 13.7 & 291 \\
PUE-PCL & 1.37 & 2.74 & 3.39 & 6.49 & 17.8 & - & - & - & - & 49.1 & 398 \\
PUE-PCD-T1meso & 2.91 & 5.17 & 7.39 & 18.7 & 56.3 & - & - & - & - & 63.5 & 312 \\
PUE-PCD-T2meso & 3.42 & 5.89 & 8.54 & 19.8 & 44.3 & - & - & - & - & 61.8 & 340 \\
PUE-PCD-T3meso & 4.20 & 7.40 & 10.5 & 20.8 & 39.2 & - & - & - & - & 49.4 & 341 \\
PUE-PCD-T4meso & 4.81 & 8.23 & 11.4 & 21.3 & 35.3 & - & - & - & - & 38.2 & 320 \\
PUE-PCD-T5meso & 4.93 & 8.85 & 12.2 & - & - & - & - & - & - & 29.1 & 266 \\
PUE-PCD & 1.25 & 2.87 & 3.95 & 11.9 & - & - & - & - & - & 58.9 & 273 \\
\hline
\end{tabular}

${ }^{\mathrm{a}}$ Tensile properties measured at room temperature $\left(23^{\circ} \mathrm{C} \pm 2^{\circ} \mathrm{C}\right)$ with strain speed of $100 \mathrm{~mm} / \mathrm{min}$. ${ }^{\mathrm{b}} \mathrm{Tensile} \mathrm{strength} \mathrm{at} \mathrm{breaking} \mathrm{point.}{ }^{\mathrm{c}}$ Elongation at breaking point. 


\subsection{Thermal Properties}

In Tables 2-4, one main transition of the composites is observed in the DSC scans in the range from $-100^{\circ} \mathrm{C}$ to $200^{\circ} \mathrm{C}$. The values of the glass transition temperature $\left(T_{\mathrm{g}}\right)$ for the composites are $-67.0^{\circ} \mathrm{C}$ for PUE-PTMG, $-45^{\circ} \mathrm{C}$ for PUE-PCL, and $-26.4^{\circ} \mathrm{C}$ for PUE-PCD. The values of $T_{\mathrm{g}}$ for the tartaric-acid-containing PUEs do not increase with increasing tartaric acid content. The $T_{\mathrm{g}}$ values of the PUEs with tartaric acid significantly fall in comparison with those of the PUEs without tartaric acid.

The thermal stability of the tartaric-acid-containing PUEs was examined by TGA under an $\mathrm{N}_{2}$ atmosphere. In Tables 2-4, the $10 \mathrm{wt} \%$ weight loss temperature $\left(T_{10}\right)$ for all samples decreases with increasing tartaric acid content. The $T_{10}$ values of PUE-PCL-T1-5 (L/D/meso) and PUE-PCD-T1-5 (L/D/meso) are greater than those of PUE-PCL and PUE-PCD, respectively. There were two distinct stages of decomposition. In the first stage, PUEs decomposed slowly until $300^{\circ} \mathrm{C}$, which accounted for the first $10 \%$ of the weight loss. Weight loss was very rapid in the temperature range from $300^{\circ} \mathrm{C}$ to $500^{\circ} \mathrm{C}$. The main degradation process can be observed at temperatures around $450^{\circ} \mathrm{C}$. The weight loss of the PUEs containing tartaric acid increases when the hard segment content (tartaric acid) increases, a fact which is in accordance with the existence of a higher number of weaker urethane bonds. The first stage of degradation is dominated by urethane bond decomposition.

DMA of the tartaric-acid-containing PUEs was carried out in temperature range from $-100^{\circ} \mathrm{C}$ to $300^{\circ} \mathrm{C}$. The results are shown in Tables $2-4$. The rubber flat regions of the PUEs with tartaric acid are unaltered for all samples. The peak positions of $\tan \delta$ for the PUEs with tartaric acid are unaltered for all samples. However, in the cases of PUE-PTMG-T1-5 (L/D/meso) and PUE-PCD-T1-5 (L/D/meso), the peaks broaden with increasing tartaric acid content.

\subsection{Optical Rotation}

The specific optical rotations of the chiral tartaric-acid-containing PUEs are shown in Tables 2-4. Optical rotation of the $\mathrm{L}(+)$-tartaric-acid-containing PUEs occurs in the positive direction. Conversely, optical rotation of the $\mathrm{D}(-)$-tartaric-acid-containing PUEs occurs in the negative direction. The PUEs containing meso-tartaric acid do not exhibit any optical rotation. In addition, the specific optical rotations of the tartaric-acid-containing PUEs increase with increasing tartaric acid content. These results suggest that the asymmetric center remains in the main chains of PUEs containing $\mathrm{L}(+)$ - or D(-)-tartaric acid.

\subsection{Molecular and Higher-Order Structures of Chiral Polyurethane Elastomers Containing Tartaric Acid}

The reactivity of the NCO group towards highly acidic functional groups is very low. Therefore, a reaction between the carboxyl group in tartaric acid and the NCO group would not be expected. The molecular structures of PUE and the tartaric-acid-containing PUEs were predicted using Gaussian molecular calculations. The results are shown in Figure 5. Two carboxyl groups in tartaric acid are assigned to outside of the molecular chain. Thus, an intermolecular hydrogen bond to another molecular chain would be formed. Next, the effect of tartaric acid on the high-order structure of PUE was investigated. The chiral PUE containing tartaric acid is predicted to form a para-helix structure. The L(+)-tartaric-acid-containing PUE would form a right-handed para-helix structure, while the $\mathrm{D}(-)$-tartaric-acid-containing PUE would form a left-handed para-helix structure. Intramolecular hydrogen bonds are formed between para-helix molecular chains. The carboxyl group in tartaric acid is expected to be located outside of the para-helix molecular chain. The intermolecular hydrogen bonding was observed to increase with increasing tartaric acid content. Therefore, chiral PUEs with tartaric acids calcified as the tartaric acid content increased. However, when an appropriate amount of tartaric acid was added, the strengths and flexibilities of PUEs with tartaric acid increased.

\section{Conclusion}

In the present work, a series of chiral PUEs containing tartaric acid was synthesized from chiral tartaric acid, polyether or polyester polyol, and diisocyanate by a one-shot method. Sheets of the colorless translucent tartaric-acid-containing PUEs were obtained when PTMG2000 was used as the polyol, while translucent light-green and yellow sheets were obtained using PCL2000 and PCD2000, respectively. The sheets showed solvent resistances similar to that of the mother polyurethane due to the formation of network structures. They changed from 
I PUE-PTMG-TL

PUE with introducing of $\mathrm{L}(+)$-Tartaric acid
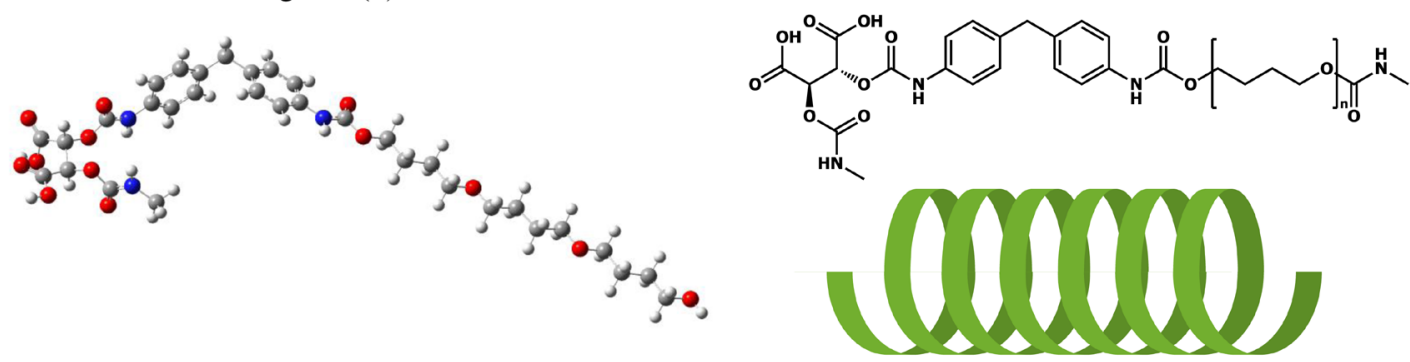

\section{- PUE-PTMG-TD}

PUE with introducing of D(-)-Tartaric acid
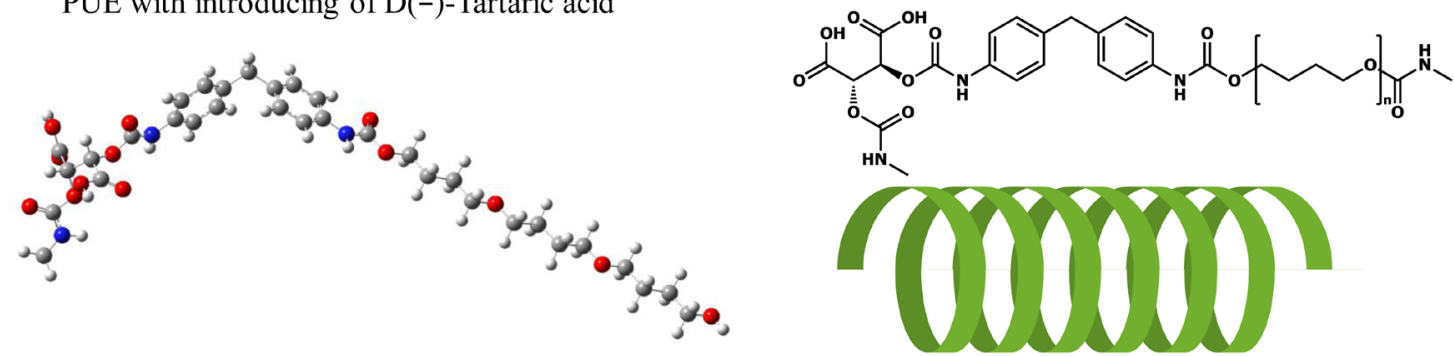

Figure 5. One of possible structures of the chiral PUE containing $\mathrm{L}(+)-$ and $\mathrm{D}(-)$ - tartaric acid.

elastic (PUE-(PTMG/PCL/PCD)-T1 and 2 (L/D/meso)) to plastic-like (PUE-(PTMG/PCL/PCD)-T3-5 (L/D/meso)) due to the effect of an increase in tartaric acid content on the chemical structure and crosslink density. The inclusion of $0.65 \mathrm{wt} \%$ and/or $1.4 \mathrm{wt} \%$ tartaric acid improved the mechanical properties such as tensile strengths and elongation at the breaking points of the PUEs, and the sheets were elastic. The chiral PUEs containing tartaric acid had asymmetric structures. These results provide a better understanding of the relationships between the structures and properties of chiral PUEs.

\section{References}

[1] Szycher, M. (1999) Szycher’s Handbook of Polyurethanes. CRC Press, Boca Raton, 1.1-9.

[2] Wirtz, H. and Schulte, K. (1973) Processing of Polyurethane Foam Systems. Kunststoffe, 63, 726-730.

[3] Avar, G., Meier, W.U., Casselmann, H. and Achten, D. (2012) Polymer Science: A Comprehensive Reference. Polymer Science: A Comprehensive Reference, 10, 411-441.

[4] Kuehn, A.F. (1986) Polyurethane-A Promising Prospect in Roll Coverings. PIMA, 68, 27-28.

[5] Gagro, D. (2010) Polyurethanes Overview of the PUR Market. European Coatings Journal, 10, 9-11.

[6] Bez, W. and Quack, G. (1983) Polyurethane and Latex Foam Carpet Backing Today. Cellular Polymers, 2, 31-53.

[7] Hare, C.H. (2000) A Review of Polyurethanes: Formulation Variables and their Effects on Performance. Journal of Protective Coatings and Linings, 17, 34-44.

[8] Paulmann, U. (2015) Polyurethane Elastomers from Polyether Polyol Mixtures and Trimerized Diisocyanates. GER. Offen, DE 102013022173 A1 20150625.

[9] Qiu, F.-X., Wang, Q., Chen, C.-H., Zhao, H., Liu, J.-H. and Yang, D.-Y. (2013) Sunthesis and Thermos-Optic Properties of Azo Polyurethane Containing Chiral Unit. Key Engineering Materials, 538, 73-76, 5.

[10] Liu, J.-H., Qiu, F.-X., Cao, G.-R., Shen, Q., Cao, Z.-J. and Yang, D.-Y. (2011) Preparation, Thermos-Optic Property and Transmission Loss of Chiral Azobenzene Polyurethane. Journal of Applied Polymer Science, 121, 2567-2572. http://dx.doi.org/10.1002/app.33980

[11] Qiu, F.-X., Zhang, W., Liu, J.-H. and Yang, D.-Y. (2010) Optically Active Polyurethane Containing Asymmetric Cen- 
ter: Preparation, Characterization and Thermo-Optic Properties. Polymer-Plastics Technology and Engineering, 49, 1521-1526. http://dx.doi.org/10.1080/03602559.2010.512330

[12] Zhang, W., Liu, J.-H., Guan, Y.-J., Cao, G.-R., Yang, D.-Y. and Qiu, F.-X. (2010) Preparation of Novel Azo Polyurethane and the Film Thermos-Optic Property. Journal of Optoelectronics Laser, 21, 396-399.

[13] Cao, G.-R., Guan, Y.-J., Zhang, W., Liu, J.-H., Qiu, F.-X., Yang, D.-Y. and Zhou, Y.-M. (2010) Synthesis, ThermoOptic Property and Dispersion of Novel Optically Active Azo Polyurethane. Functional Materials, 41, 911-914.

[14] Qiu, F.-X., Zhang, W., Yang, D.-Y., Zhao, M.-J., Cao, G.-R. and Li, P.-P. (2010) Synthesis, Characterization, and Thermo-Optical Properties of Azobenzene Polyurethane Containing Chiral Units. Journal of Applied Polymer Science, 115, 146-151.

[15] Gudeangadi, P.G., Sakamoto, T., Shichibu, Y., Konishi, K. and Nakano, T. (2015) Chiral Polyurethane Synthesis Leading to $\pi$-Stacked 2/1-Helical Polymer and Cyclic Compounds. ACS Macro Letters, 4, 901-906.

[16] Varkey, E.C. and Sreekumar, K. (2010) Isosorbide Based Chiral Polyurethanes: Optical and Thermal Studies. Journal of Materials Science, 45, 1912-1920. http://dx.doi.org/10.1007/s10853-009-4177-1

[17] Huang, S.-H., Bai, Z.-W., Yin, C.-Q. and Li, S.-R. (2006) Covalently Bonding Chiral Polyurethane on Aminated Silica Gel: A New Strategy to Prepare Chiral Stationary Phase for High Performance Liquid Chromatography. Chinese Journal of Polymer Science, 24, 107-114. http://dx.doi.org/10.1142/S0256767906001102

[18] Lui, J.-H., Tai, F.-R. and Lai, Y.-C. (1995) Synthesis of Chiral Polymer Having Camphanediol Moieties and Their Optical Resolution of Some Racemates. Journal of Applied Polymer Science, 58, 1713-1720.

[19] Lui, J.-H., Tsai, F.-R. and Lai, Y.-C. (1995) Synthesis and Characteristics of Chiral Polymers with Camphanediol Moieties. Die Angewante Makromolekulare Chemie, 231, 35-45. http://dx.doi.org/10.1002/apmc.1995.052310104

[20] Haider, K.W., Chan, J.C., Jonsson, E.H. and Franz, U.W. (2001) Hydrophobic Polyurethane Elastomer. US 6211324 B1 20010403.

[21] Chen, C.-F., Su, Q., Chen, Y.-M. and Xi, F. (1999) A New Optically Active Polyurethane Derived from Chiral (2R, 3R) (+)-Diethyl L-Tartrate and Diisocyanate. Chinese Journal of Polymer Science, 17, 371-373.

[22] Behr, J.P., Girodeau, J.M., Hayward, R.C., Lehn, J.M. and Sauvage, J.P. (1980) Molecular Receptors. Functionalized and Chiral Macrocyclic Polyethers Derived from Tartaric acid. Helvetica Chimica Acta, 63, 2096-2111. http://dx.doi.org/10.1002/hlca.19800630736

[23] Gryko, D.T., Piatek, P. and Jurczak, J. (1999) An Efficient Method for Preparation of Chiral Macrocyclic Bisamides Starting from Diol Derivatives of D-Mannitol and L-Tartaric Acid. Synthesis, 2, 336-340. http://dx.doi.org/10.1055/s-1999-6054

[24] Li, B., Yang, X., Yang, K. and Fu, E. (2005) Synthesis of Novel Chiral Macrocyclic Polyamides Derived from L-/DTartaric Acid. Synthetic Communications, 35, 2603-2608. http://dx.doi.org/10.1080/00397910500214235

[25] Li, B., Yang, X., Wu, X., Luo, X., Zhong, C. and Fu, E. (2006) Enantioselective Recognition for Carboxylic Acids by Novel Chiral Macrocyclic Polyamides Derived from L-/D-Tartaric Acid. Supramolecular Chemistry, 18, 507-513. http://dx.doi.org/10.1080/10610270600808141

[26] Seebach, D., Crass, G., Wilka, E.M., Hilvert, D. and Brunner, E. (1979) Three New Chiral Aminoethers from Tartaric Acid for Improved Asymmetric Syntheses with Organolithium Reactions. Preliminary Communication. Helvetica Chimica Acta, 62, 2695-2698. http://dx.doi.org/10.1002/hlca.19790620819

[27] Nitta, Y., Sekine, F., Imanaka, T. and Teranishi, S. (1982) Effect of Preparation Variables on Enantioselectivity of Supported Nickel Catalysts Modified with Tartaric Acid. Journal of Catalysis, 74, 382-392. http://dx.doi.org/10.1016/0021-9517(82)90043-4

[28] Gao, J.-M., Liu, W.-T., Li, M.-L., Liu, H.-W., Zhang, X.-C. and Li, Z.-X. (2008) Preparation and Structural Elucidation of (-)-Tetrahydroberberine-(+)-2,3-Di(P-Toluyl) Tartaric Acid Complex. Journal of Molecular Structure, 892, 466-469. http://dx.doi.org/10.1016/j.molstruc.2008.06.013

[29] Kimino, T. and Fujii, I. (2010) Crystal Structure of (2R, 3R)-Tartaric Acid Complex of (R)-Nipecotic Acid Ethyl Ester. X-Ray Structure Analysis Online, 26, 11-12. http://dx.doi.org/10.2116/xraystruct.26.11

[30] Sevukarajan, M., Sodanapalli, R., Thanuja, B., and Nair, R. (2011) Preparation and Pharmaceutical Characterization of Supra Molecular Complex of Isoniazid with L(+) Tartaric Acid. Journal of Biomedical Sciences and Research, 3, 397402.

[31] Violante de Paz, M., Marín, R., Zamora, F., Hakkou, K., Alla, A., Galbis, J.A. and Muñoz-Guerra, S. (2007) Linear Polyurethanes Derived from Alditols and Diisocyanates. Journal of Polymer Science, 45, 4109-4117. http://dx.doi.org/10.1002/pola.22127

[32] Marín, R., Martínez de Ilarduya, A. and Muñoz-Guerra, S. (2009) Linear Polyurethanes Made from Naturally Occurring Tartaric Acid. Journal of Polymer Science, 47, 2391-2407. http://dx.doi.org/10.1002/pola.23330 
[33] Hatakeyama, H., Hirose, S. and Hatakeyama, T. (1995) Biodegradable Polyurethanes from Plant Components. Journal of Macromolecular Science, Part A: Pure and Applied Chemistry, 32, 743-750. http://dx.doi.org/10.1080/10601329508010285

[34] Wang, G. and Zhou, A. (2011) Soy Protein Based Biodegradable Flexible Polyurethane Foam. Advanced Materials Research, 152, 1862-1865. http://dx.doi.org/10.4028/www.scientific.net/AMR.264-265.1862

[35] Asano, Y. and Hatakeyama, H. (2003) Rigid Type Polyurethane Foams Containing Saccharide and Lignin Structures in the Molecular Chain. Memoirs of Fukui University of Technology, 33, 275-282.

[36] Hatakeyama, H. and Hatakeyama, T. (2005) Environmentally Compatible Hybrid-Type Polyurethane Foams Containing Saccharide and Lignin Components. Macromolecular Symposia, 224, 219-226. http://dx.doi.org/10.1002/masy.200550619

[37] Chen, Q., Li, R., Sun, K., Li, J. and Liu, C. (2011) Preparation of Bio-Degradable Polyurethane Foams from Liquefied Wheat Straw. Advanced Materials Research, 217-218, 1239-1244. http://dx.doi.org/10.4028/www.scientific.net/amr.217-218.1239

[38] Garcon, R., Clerk, C., Gesson, J.-P., Bordado, J., Nunes, T., Caroco, S., Gomes, P.T., Minas da Piedade, M.E. and Rauter, A.P. (2001) Synthesis of Novel Polyurethanes from Sugars and 1,6-Hexamethylene Diisocyanate. Carbohydrate Polymers, 45, 123-127. http://dx.doi.org/10.1016/S0144-8617(00)00323-4

[39] Lim, H., Kim, E.Y. and Kim, B.K. (2010) Polyurethane Foams Blown with Various Types of Environmentally Friendly Blowing Agents. Plastics, Rubber and Composites, 39, 364-369. http://dx.doi.org/10.1179/174328910X12691245469835

[40] Ionescu, M., Mihalache, I., Zugravu, V. and Mihai, S. (1994) Inherently Flame Retardant Rigid Polyurethane Foams Based on New Triazinic Polyether Polyols. Cellular Polymers, 13, 57-68. 